\title{
THE
}

\section{Examination of the Necessity of Complete Wetting near Critical Points in Systems with Long-Range Forces}

\author{
M. P. Nightingale \\ University of Rhode Island, nightingale@uri.edu \\ J. O. Indekeu
}

Follow this and additional works at: https://digitalcommons.uri.edu/phys_facpubs

Terms of Use

All rights reserved under copyright.

\section{Citation/Publisher Attribution}

Nightingale, M. P., \& Indekeu, J. O. (1985). Examination of the necessity of complete wetting near critical points in systems with long-range forces. Physical Review B, 32(5), 3364-3366. doi: 10.1103/

PhysRevB.32.3364

Available at: http://dx.doi.org/10.1103/PhysRevB.32.3364

This Article is brought to you for free and open access by the Physics at DigitalCommons@URI. It has been accepted for inclusion in Physics Faculty Publications by an authorized administrator of DigitalCommons@URI. For more information, please contact digitalcommons-group@uri.edu. 


\title{
Examination of the necessity of complete wetting near critical points in systems with long-range forces
}

\author{
M. P. Nightingale \\ Department of Physics, University of Rhode Island, Kingston, Rhode Island 02881 and Department of Physics, \\ Massachusetts Institute of Technology, Cambridge, Massachusetts 02139 \\ J. O. Indekeu \\ Instituut voor Theoretische Fysica, Katholieke Universiteit Leuven, B-3030 Leuven, Belgium \\ and Department of Physics, Massachusetts Institute of Technology, \\ Cambridge, Massachusetts 02139 \\ (Received 28 May 1985)
}

\begin{abstract}
Cahn's general argument for complete wetting in the vicinity of critical points is critically reviewed. Critical-point wetting does occur in systems with short-range (exponentially decaying) forces. Whenever short-range forces favor wetting, while at the same time there is a tendency towards drying due to weak long-range (algebraically decaying) forces, neither critical-point wetting nor drying takes place. In this case the thickness of the partial wetting layer diverges as the bulk correlation length upon approach of the critical point.
\end{abstract}

The purpose of this paper is to investigate the general validity of the arguments, presented originally by Cahn, ${ }^{1}$ that indicate that complete wetting always occurs in the vicinity of critical points: critical-point wetting. The main results are the following. In systems with short-range forces we do, as predicted by Cahn, find critical-point wetting. However, the presence of forces of long range (in the sense of algebraic decay at large distances) may inhibit a wetting transition. These forces generate effective interactions between interfaces ${ }^{2}$ that can, together with opposing shortrange interactions, prevent complete wetting. Under these circumstances, one has a partial wetting layer of thickness proportional to the bulk correlating length, so that the critical point itself is a point of continuous wetting.

In a container with two coexisting phases $\alpha$ and $\beta$, the wall in general will preferentially adsorb one of the two phases, say $\alpha$. This phase completely wets the wall if a macroscopically thick layer of $\alpha$ spontaneously intrudes between $\beta$ and the wall. On the other hand, if only a microscopically thin film of the $\alpha$ phase forms at the wall, this is called incomplete wetting. ${ }^{3}$ In terms of the contact angle $\theta$ of a macroscopic droplet of the $\alpha$ phase in contact with the wall, $0<\theta<\pi$ is incomplete wetting, and $\theta=0$ is complete wetting. If it is necessary to make the distinction, the term drying is used, where the roles of $\alpha$ and $\beta$ are interchanged, and $\theta \rightarrow \pi-\theta$.

The preferential adsorption of one of the phases at the wall can be modeled in terms of a local field $h_{1}$ (used in the general sense) acting strictly at the wall. Let $\alpha$ be favored for $h_{1}>0$, and $\beta$ for $h_{1}<0$. Now imagine an infinite container and consider the surface layer bounded by the wall on one side, and by the $\alpha$ bulk phase infinitely far away on the other side. The surface tension associated with the wall under these conditions is denoted $\sigma_{\alpha}$; likewise, $\sigma_{\beta}$ is defined as the surface tension if the bulk phase is $\beta$. For small boundary fields $h_{1}$, and if the system is sufficiently far away from the critical point (in a sense to be made more precise below), the surface layer will not qualitatively differ from the case $h_{1}=0$, as illustrated in Fig. 1. One can then attempt to expand

$$
\sigma_{\alpha}\left(h_{1}\right)=\sigma_{0}-h_{1} m_{1}
$$

and

$$
\sigma_{\beta}\left(h_{1}\right) \simeq \sigma_{0}+h_{1} m_{1},
$$

where $m_{1}$ is the absolute value of the density conjugate to $h_{1}$, i.e., the surface order parameter. For simplicity, we have assumed symmetry with respect to interchange of the two phases. Let $m$ be the bulk order parameter which takes on the value $m_{\alpha}$ and $m_{\beta}$ in the $\alpha$ and $\beta$ phases, respectively. For small fields $h_{1}$, typical profiles of the density versus $z$, the distance from the wall, are shown in Fig. 1. Of course, even for $h_{1}=0$ (dashed curve) the order parameter in the bulk deviates from its magnitude at the wall, where the ordering generally is weaker.

Let $T_{c}$ be the critical temperature of the system. Here the $\alpha$ and $\beta$ phases become identical. The temperature deviation away from the critical point is denoted $t=\left(T-T_{c}\right) / T_{c}$. For small $t$ one has

$$
\sigma_{\beta}=\sigma_{\alpha}+2 h_{1} m_{1}=\sigma_{\alpha}+B_{1} h_{1}|t|^{\beta_{1}},
$$

where $\beta_{1}$ is the critical exponent that governs the vanishing, as a function of temperature, of the surface order parameter for $h_{1}=0 ; B_{1}>0$ is a constant. Note that Eq. (2) features

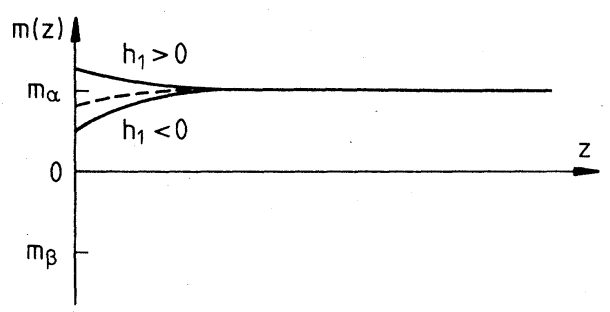

FIG. 1. Order parameter $m$ vs distance $z$ of the wall- $\alpha$ surface for small surface fields $h_{1}>0, h_{1}=0$ (dashed line), and $h_{1}<0$. 
the surface critical exponent $\beta_{1}$ (Ref. 4) in contrast to the earlier literature, ${ }^{1}$ where it is assumed that $\sigma_{\alpha}-\sigma_{\beta}$ is proportional to the bulk order parameter close to the critical point, and thus would behave as $|t|^{\beta}$. Under a spatial rescaling of the system with a scale factor $b$, one has $t \rightarrow b^{y_{\mathrm{T}}} t$, $y_{\mathrm{T}}$ being the thermal critical exponent, and further $h_{1} \rightarrow b^{y_{\mathrm{H}}^{\prime}} h_{1}$, with $y_{\mathrm{H}}^{\prime}$ the surface field exponent. ${ }^{5}$ In terms of these exponents, the singular part of the surface tension $\sigma_{\alpha, \text { sing }}$ satisfies the scaling equation

$$
\sigma_{\alpha, \text { sing }}\left(t, h_{1}\right)=b^{1-d} \sigma_{\alpha, \text { sing }}\left(b^{y_{\mathrm{T}}} t, b^{y_{\mathrm{H}}^{\prime}} h_{1}\right),
$$

assuming a $d-1$ dimensional surface of a $d$ dimensional system. If follows immediately that $\beta_{1}=\left(d-1-y_{\mathrm{H}}^{\prime}\right) / y_{\mathrm{T}}$.

The interface between the $\alpha$ and $\beta$ phases has an interface tension, denoted by $\sigma_{\alpha \beta}$, which satisfies the inequality

$$
\left|\sigma_{\alpha}-\sigma_{\beta}\right| \leqslant \sigma_{\alpha \beta}
$$

Namely, suppose to the contrary that e.g., $\sigma_{\beta}>\sigma_{\alpha \beta}+\sigma_{\alpha}$. Under these circumstances, as the usual argument goes, the wall- $\beta$ profile would be unstable against the formation of a compound structure consisting of the conjunction of a wall$\alpha$ surface and an $\alpha-\beta$ interface at infinity.

On approach of the critical point the $\alpha-\beta$ interface tension vanishes as

$$
\sigma_{\alpha \beta} \simeq M|t|^{i \mu},
$$

where $M$ is a positive amplitude and $\mu=(d-1) / y_{\mathrm{T}}$, which follows immediately from the fact that the interface tension satisfies an equation of the same form as Eq. (3), with $h_{1}=0$. The relation for $\beta_{1}$ given above immediately yields $\beta_{1}<\mu$ since the surface field is a relevant variable (in the renormalization group sense), i.e., $y_{\mathrm{H}}^{\prime}>0$. As a consequence, $\sigma_{\alpha \beta}$ vanishes more rapidly than $\left|\sigma_{\alpha}-\sigma_{\beta}\right|$ as given by Eq. (2), so that from Eq. (4) it follows that Eq. (2) breaks down if

$$
B_{1} h_{1}>M|t|^{y_{\mathrm{H}}^{\prime} / y_{\mathrm{T}}} \text {. }
$$

In other words, for any small, but fixed value of $h_{1}$, Eq. (6) defines a temperature region around the critical point, where the order-parameter profile undergoes a qualitative change. It differs from the profile for $h_{1}=0$ by more than just the small perturbation assumed in Eq. (2). In this region, if the wall preferentially adsorbs the $\alpha$ phase, the sum of the surface free energies of a wall- $\alpha$ surface and an $\alpha-\beta$ interface is less than the surface tension $\sigma_{\beta}$ as given by Eq. (2). One might conclude that where Eq. (6) holds there is complete wetting. However, this conclusion is warranted only if there are no other, partial wetting configurations which have a lower free energy yet.

A potential candidate of the latter is the following. The thermodynamically stable profile in the regime defined by Eq. (6) could in principle consist of a wall- $\alpha$ surface and an $\alpha-\beta$ interface at a finite (microscopic) rather than at an infinite (macroscopic) distance $\zeta$ from the wall, as illustrated in Fig. 2. Such a structure is physically well defined as long as $\zeta$ is greater than the width of the core of the $\alpha-\beta$ interface, which typically is of order $\xi$. In this case, let us write

$$
\sigma_{\beta}(\zeta)=\sigma_{\alpha}+\sigma_{\alpha \beta}+\Delta f(\zeta),
$$

where $\Delta f(\zeta)<0$ represents the effective interaction between the wall- $\alpha$ surface and the $\alpha-\beta$ interface at a separation $\zeta$. The crossover between the regimes where
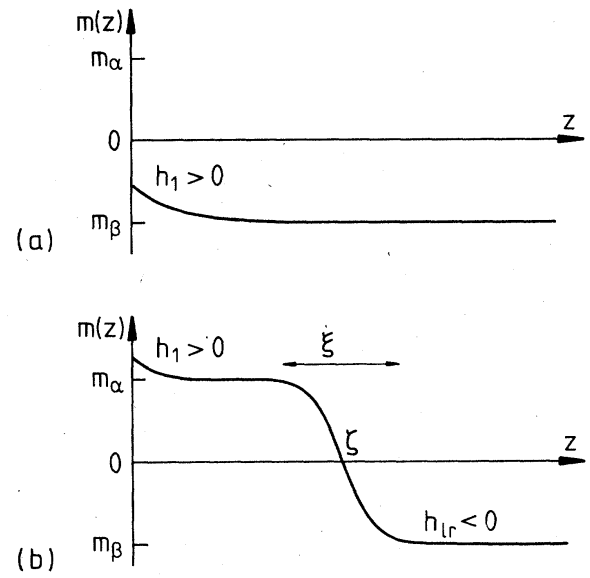

FIG. 2. (a) The wall- $\beta$ profile changes over to (b) a combination of a wall- $\alpha$ profile and an $\alpha-\beta$ interface at a finite distance $\zeta>\xi$.

Eqs. (2) and (7) are appropriate is possible without any phase transition whatsoever. However, a (generically firstorder) thin-film to thick-film phase transition might occur. Whether a transition to complete wetting occurs or not depends on the behavior of the microscopic interactions at large distances, as we shall now discuss in detail.

Let us explicitly consider a system with long-range forces where neither critical-point wetting nor drying takes place. In a phenomenological description of the interactions of the system with the wall we introduce two fields. In the first place, there is the surface field $h_{1}$, as before. It describes the interactions in range of a few $\AA$. Secondly, there is a field $h_{\mathrm{lr}}$, that describes the net effect of the long-range forces. It has an algebraic dependence on distance: $h_{\mathrm{lr}}(z)=a z^{-\omega}$, for $z>\xi$. For van der Waals forces $\omega=3$ (nonretarded; thin films) or $\omega=4$ (retarded; thick films). Suppose that $h_{\mathrm{lr}}<0$, so that the long-range field favors the $\beta$ phase, and at the same time $h_{1}>0$, favoring the $\alpha$ phase close to the wall. Moreover, we suppose that $h_{1 \mathrm{r}}$ is sufficiently weak that Eq. (2) holds far from the critical point, so that $\sigma_{\alpha}<\sigma_{\beta}{ }^{6}$ Now consider an $\alpha-\beta$ interface at a distance $\zeta$ from the wall, as illustrated in Fig. 2(b). Due to the presence of $h_{\mathrm{lr}}$ the $\alpha-\beta$-like interface has an interface tension lower than at infinite distance from the wall by an amount of order

$$
\int_{\zeta}^{\infty} a z^{-\omega} m d z \sim a m \zeta^{1-\omega}
$$

where $m$ is the absolute value of the bulk order parameter. In fact, due to the long-range forces, the interface develops algebraically decaying tails at distances much larger than its width which is of order $\xi$. This effect contributes to the surface tension by an amount negligable compared to the contribution given in Eq. (8).

Due to the presence of the long-range field $h_{\text {Ir }}$ the interface will be attracted to the wall. However, at distances closer to the wall than of order $\xi$, appreciable distortion of the interface sets in. This results in an effective repulsion and an increase of surface free energy of order $e^{-\zeta / \xi}$. We conclude that in equilibrium the $\alpha-\beta$ interface will be at a distance from the wall greater than, but comparable to, the bulk correlation length. The corresponding decrease in surface free energy $\Delta f$ will be of order $a m \xi^{1-\omega}$. As a conse- 
quence, incomplete wetting persists all the way up to the critical point. Here, complete wetting takes place continuously with an $\alpha$-phase wetting layer that diverges in thickness like the bulk correlation length.

If only short-range (exponentially decaying) forces are present, the effective repulsion of order $e^{-\zeta / \xi}$ will ultimately dominate upon approach of the critical point. In this case there is critical-point wetting.

Of course, only as long as $\sigma_{\alpha}<\sigma_{\beta}$, does it make sense to consider the question whether $\alpha$ wets the wall. That is, as soon as this condition, which via Eq. (7) is equivalent to $-\Delta f(\zeta)>\sigma_{\alpha \beta}$, is violated we would have to consider the possibility of complete drying. Indeed, there are two possible ways in which this may happen. In the first place, the amplitude $a$ of the long-range field $h_{\text {Ir }}$ could be sufficiently big so as to change the nature of the wall, i.e., causing preferential adsorption of $\beta$ rather than $\alpha$. In this case, drying will result as there are no opposing long-range forces. The other possibility is that the amplitude $a$ is arbitrarily small but the algebraic decay of the field is too slow. Then the stability breaks down of the profile, as shown in Fig. 3(a), connecting the wall with a bulk phase $\alpha$. Indeed, as soon as $-\Delta f(\xi)>2 \sigma_{\alpha \beta}$, this profile is unstable against the formation of two $\alpha-\beta$ interfaces. Again a $\beta$-phase drying layer, excluding $\alpha$ from contact with the wall, is precipitated, as shown in Fig. 3(b). Clearly, the condition to avoid this instability is $\left|a m \xi^{1-\omega}\right| \leq \sigma_{\alpha \beta}$, i.e., $\omega>y_{\mathrm{H}}$, using $\xi \sim|t|^{-1 / y_{\mathrm{T}}}$ and $m \sim|t|^{\beta}$, with $\beta=\left(d-y_{\mathrm{H}}\right) / y_{\mathrm{T}}$. For van der Waals forces the condition $\omega>y_{\mathrm{H}}$ is satisfied: $\omega=3$ or 4 , and $y_{\mathrm{H}}<3$ for continuous transitions in three dimensions.

Remark that the condition that $\omega>y_{\mathrm{H}}$ is required in order that the thickness of the wetting layer diverge with $\xi \sim|t|^{-1 / y_{\mathrm{T}}}$. That is, at the $\alpha-\beta$ interface there is an ordering field of order $h_{\mathrm{lr}}(\xi)$. To obtain the temperaturelike divergence of $\xi$ nevertheless, the requirement is $h_{\mathrm{lr}}^{1 / y_{\mathrm{H}}}$ $=O\left(|t|^{1 / y_{\mathrm{T}}}\right)$ which at once yields $\omega>y_{\mathrm{H}}$. This follows from the scaling form of the correlation length: $\xi=|t|^{-1 / y_{\mathrm{T}}} X\left(h|t|^{-y_{\mathrm{H}} / y_{\mathrm{T}}}\right)$, where $X$ can be Taylor expanded yielding $|t|^{-1 / y_{\mathrm{T}}}$ as the dominant singularity, as long as $|h|^{1 / y_{\mathrm{H}}}<<|t|^{1 / y_{\mathrm{T}}}$.

The competition between short- and long-range forces that may suppress critical-point wetting can be studied experimentally. Dixon, Schlossman, Wu, and Franck have studied adsorption profiles of a critical binary mixture of nitromethane and carbon disulfide on differently prepared borosilicate glass substrates. ${ }^{7}$ In terms of the surface field $h_{1}$, the preparation of the surface determines its sign. A hydroxylated, polar surface preferentially adsorbs the polar nitromethane molecules at short range. This we choose to

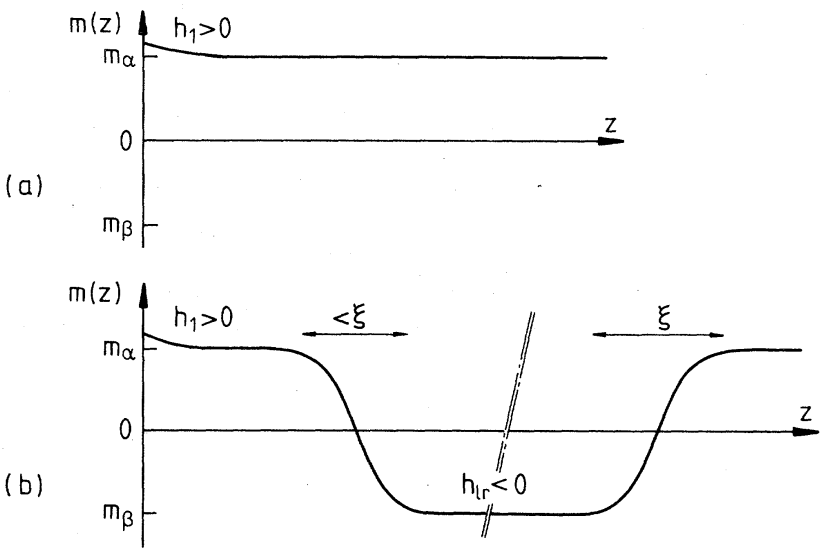

FIG. 3. (a) A wall- $\alpha$ interface becomes unstable and (b) two $\alpha-\beta$ interfaces spontaneously form: one at $z \geq \xi$ and the other at infinity.

identify with $h_{1}<0$. The methylated substrate shows the opposite behavior, i.e., it favors carbondisulfide: $h_{1}>0$. At large distances, irrespective of the surface preparation, the long-range forces always lead to a greater relative adsorption of nitromethane: $h_{\mathrm{lr}}<0$. Indeed, in very recent experiments it has been observed that in the case of a methylated substrate, complete wetting does not occur before the critical point is reached. ${ }^{8}$

Finally, remark that there exist long-range forces of cooperative, rather than of microscopic nature. ${ }^{9}$ In addition to the van der Waals forces referred to so far, cooperative long-range forces are present in a partial wetting layer under thermodynamic conditions such that an infinitely thick layer would be critical. The incipient long-range correlations in such a near-critical wetting layer give rise to a long-range potential which depends on its thickness $z$ as $z^{-2}$, with an amplitude typically of the same order as, or greater than, that due to van der Waals forces. ${ }^{10}$

It is a great pleasure to thank C. Franck for his stimulating explanation of the wetting experiments mentioned above. This work was supported in part by the National Science Foundation under Grant No. DMR-84-06186 at the University of Rhode Island and by the U.S. Joint Services Electronics Program under Contract No. DAAG29-83K0003 at MIT. J.O.I. thanks the Council for International Exchange of Scholars and the Belgian National Fund for Scientific Research for financial support. He also acknowledges A. N. Berker's support and hospitality at MIT.
1J. W. Cahn, J. Chem. Phys. 66, 3667 (1977); M. R. Moldover and J. W. Cahn, Science 207, 1073 (1980).

2P.-G. de Gennes, J. Phys. (Paris) Lett. 42, L377 (1981).

${ }^{3}$ For recent reviews, see, e.g., M. R. Moldover and J. W. Schmidt, Physica D 12, 351 (1984); P.-G de Gennes, Rev. Mod. Phys. (to be published).

${ }^{4}$ R. Pandit, M. Schick, and M. Wortis, Phys. Rev. B 26, 5112 (1982).

${ }^{5}$ N. M. Ś Srakić and M. Wortis, Phys. Rev. B 15, 396 (1977); N. M. Švrakić, R. Pandit, and M. Wortis, ibid. 22, 1286 (1980).

${ }^{6}$ As long as $y_{\mathrm{H}}-y_{\mathrm{H}}^{\prime}<\omega$, where $y_{\mathrm{H}}$ is the critical exponent of the bulk ordering field, the presence of $h_{1 \mathrm{r}}$ leads to a shift of $h_{1}$ in
Eq. (2) by an amount proportional to $a$. If $\omega<y_{\mathrm{H}}-y_{\mathrm{H}}^{\prime}$, there will be an additional term in Eq. (2) proportional to $a|t|^{\beta+(\omega-1) \nu}$, where $\nu=1 / y_{\mathrm{T}}$.

${ }^{7}$ J. A. Dixon, M. Schlossman, X.-L. Wu, and C. Franck, Phys. Rev. B 31, 1509 (1985).

${ }^{8}$ C. Franck (private communication); K. Abeyaratne and C. Franck, Bull. Am. Phys. Soc. 30, 556 (1985).

${ }^{9}$ M. E. Fisher, and P.-G. de Gennes, C. R. Acad. Sci. 287, 207 (1978).

${ }^{10}$ M. P. Nightingale and J. O. Indekeu, Phys. Rev. Lett. 54, 1824 (1985). 